\title{
Radiation-induced breast angiosarcoma: report of two patients after accelerated partial breast irradiation (APBI) and review of the literature
}

\author{
Salvatore Cozzi ${ }^{1}{ }^{1}$, Masoumeh Najafi, ${ }^{2}$ Lilia Bardoscia ${ }^{1}$, Maria Paola Ruggieri ${ }^{1}$, Lucia Giaccherini ${ }^{1}$, \\ Gladys Blandino ${ }^{1}$, Andrea Botti ${ }^{3}$, Patrizia Ciammella ${ }^{1}$, Cinzia Iotti ${ }^{1}$ \\ ${ }^{1}$ Radiation Therapy Unit, Azienda USL-IRCCS di Reggio Emilia, Italy \\ ${ }^{2}$ Department of Radiation Oncology Shohadaye Haft-e-Tir Hospital, Iran University of Medical Science, Teheran, Iran \\ ${ }^{3}$ Medical Physics Unit, Department of Oncology and Advanced Technology, AUSL-IRCCS, Reggio Emilia, Italy
}

\begin{abstract}
Background: Angiosarcoma may rarely complicate radiotherapy of breast cancer. This so-called radiation-induced angiosarcoma (RIAS) occurs in less than $0.3 \%$ of patients that underwent breast conservation surgeries, usually years after completion of radiotherapy.

Case presentation: we introduce two cases of invasive ductal carcinoma who underwent lumpectomy and accelerated partial breast irradiation (APBI) as an alternative protocol to whole breast irradiation (WBI). They received adjuvant partial breast radiotherapy on tumor cavity for a total dose of 38.5 Gy in 10 fractions in 5 days using 3D-external-beam RT. In both cases, RIAS occurred eight years after radiotherapy, in the sub-cicatricial area in one patient and outside the irradiated area in the other one. They both underwent radical surgery and chemotherapy was performed in one patient.

Discussion: The underlying mechanism for development of RIAS is not well known, but its incidence seems to be increasing. RIAS after partial breast irradiation is very rare and has been reported in two cases so far. As it may be suggested in case 2 , it is still a matter of debate if the risk of radiation-induced sarcoma is radiation-dose dependent. Although mastectomy is considered as a standard treatment, choice of treatment should be made according to the patient's specifications.

Conclusion: There are very few studies in the literature that report RIAS after APBI. Present study is the only one reporting two cases after the external 3D technique APBI. Prognosis of RIAS remains poor. Only a careful evaluation in a multidisciplinary context can offer to the patients the best result in terms of local control and survival.

Key words: breast angiosarcoma; RIAS; induced-radiation angiosarcoma; APBI; late toxicities; radiotherapy; accelerated partial breast irradiation

Rep Pract Oncol Radiother 2021;26(5):827-832
\end{abstract}

\section{Introduction}

Accelerated partial breast irradiation (APBI) is an alternative protocol to whole breast irradiation (WBI) in which treatment is performed only in a restricted area of breast tissue surrounding the tumor bed. APBI delivers a higher dose of radiation to a smaller breast tissue consisting of the lumpectomy bed and a margin during a short period of time (few days versus several weeks in WBRT) [1,

Address for correspondence: Cozzi Salvatore, MD, Radiation Therapy Unit, Azienda USL-IRCCS di Reggio Emilia, Italy, Viale Risorgimento 80-42123 Reggio Emilia (RE); e-mail: salvatore.cozzi@hotmail.it; salvatore.cozzi@ausl.re.it 
2]. Several studies report a non-inferiority of APBI compared to WBRT in terms of local control and overall survival $[3,4]$ and the use of this technique is gaining more and more importance in clinical practice.

Radiotherapy for breast cancer may be complicated by radiation-induced angiosarcoma (RIAS). RIAS is a late toxicity that occurs in 0.05 to $0.3 \%$ of patients of breast cancer who underwent breast-conserving-surgery and adjuvant radiotherapy. It seems that its incidence has been increasing in recent years mirroring the increasing use of adjuvant radiation after breast conserving surgery (BCS) and its long latency time [5]. Despite its rarity, RIAS is usually described to occur nearly 10 years after breast irradiation. However, time intervals between 6 months up to 23 years have been reported in literature $[6,7]$. A recent review of Surveillance, Epidemiology, and End Results (SEER) data showed a 9-fold increased risk of breast and chest wall RIAS after breast cancer irradiation [8]. Treatment of RIAS is a matter of controversy. Surgery is considered the standard of treatment and may consist in tumor resection or mastectomy [8]. A population-based study performed in 2019 reported that an overall survival benefit of adding radiotherapy to surgery could not be confirmed [9].

Due to its rarity, information about physiopathological or radiobiological mechanisms underlying the development of RIAS are still lacking, with subsequent absence of clear, international recommendations on management and/or prevention.

We reported our experience of multidisciplinary management of two cases of RIAS secondary to breast-conserving surgery and APBI for early breast cancer.

\section{Cases presentation}

\section{Case 1}

F.C: Seventy-one-year-old woman with histologically confirmed invasive, ductal carcinoma of the right breast, grade 2, who underwent central lumpectomy and sentinel lymph nodes dissection, Luminal A cancer, pT1cNmic, negative surgical margins (R0), M0. Then, she received adjuvant partial breast radiotherapy of the tumor cavity for a total dose of 38.5 Gy in 10 fractions (3.85 Gy, twice daily, with daily interfractions time interval of at least 6 hours), target volume delineated as the site of lumpectomy plus the surrounding tissue. Adjuvant hormonal therapy with Aromatase inhibitor was also prescribed for five years. At the end of radiotherapy, the patient started regular follow up at our Breast Unit. Annual mammography and complete clinical examination every 6 months were performed for the first two years, annually thereafter, with no clinical or radiologic evidence of disease recurrence occurring in the next years.

Eight years after APBI, a sub-cicatricial lump was identified. A subsequent ultrasound (US) examination, identified a $40 \mathrm{~mm}$ nodule beyond the nipple. Trucut biopsy confirmed histological diagnosis of angiosarcoma. No pathological lymph-nodes or metastasis were found at CT-scan and PET-CT restaging. Finally, ipsilateral mastectomy was performed after patient's interview with a collegial, careful evaluation of risks and benefits, surgical and oncological outcomes deriving from a aggressive versus conservative therapeutic approach. Pathological specimen reported a 6-cm-large cutaneous breast angiosarcoma, with negative surgical margins. In view of the complete surgical excision of the lesion, taking into account the histological characterization, the previous treatments and patient's older age, adjuvant treatment has not been proposed. Actually patient is pending follow up PET TC scan restaging.

\section{Case 2}

B.M.P: Sixty-nine-year-old woman after upper external lumpectomy and sentinel lymph node biopsy for the right breast, grade 2, invasive ductal carcinoma, Luminal A cancer, pT1bN0M0R0. Adjuvant partial breast radiotherapy of the tumor cavity, with a total target dose of 38.5 Gy in 10 twice-daily fractions $(3.85 \mathrm{~Gy} /$ fraction, a minimum of 6 hours between daily fractions). Adjuvant hormonal therapy with Aromatase inhibitor for five years was also prescribed.

At the end of the radiation treatment, the patients started a regular follow up at our Breast Unit. Annual mammography plus Breast US and complete clinical examination every 6 months were performed for the first two years, annually thereafter. No clinical or radiologic evidence of disease recurrence was evident during follow up. Eight years after the end of partial radiotherapy treatment, when the patient was 77 years old, solid skin lesions quickly appeared in the inner quadrants of the right, resid- 
ual breast. There was evidence of widespread skin thickening at the subsequent mammography, with the presence of multiple, solid, cutaneous and subcutaneous nodules. Fine needle aspiration biopsy confirmed histological diagnosis of angiosarcoma. Such breast tumor masses grew quickly after the onset until they also reached the ipsilateral, outer quadrants. All this considered, following multidisciplinary discussion of the case at our Institutional Breast Tumor Board, right mastectomy was performed. The final histological exam reported a Grade 3, multifocal angiosarcoma involving the skin and residual post-lumpectpmy mammary parenchyma. Docetaxel and gemcitabine were administered as a systemic treatment because of cardiological comorbidities in which the use of anthracyclines is contraindicated. Unfortunately, systemic treatment was stopped after the first two cycles for Grade 3 gastrointestinal toxicity. Six months after surgery, the patient developed multiple pulmonary and pleural metastases and massive pleural effusion that resulted in her death one year later surgery.

\section{Radiotherapy characteristics}

In all treatments, including both cases, 3D external beam radiation was the technique used to perform APBI, according to the internal study protocol. The recommended technique consists of 4 fields: 2 tangential coplanar and two non-coplanar high-energy X photons (6 MV), with possible homogenization of the dose distribution by wedge filters. At least $90 \%$ of the PTV was to receive $>90 \%$ of the prescribed dose. The following OARs dose constrains were considered: 1) the limiting dose to the skin should not exceed $90 \%$ of the prescribed dose (PD), 2) ipsilateral breast: $<60 \%$ breast should receive $>50 \%$ of the $\mathrm{PD}$ and $<35 \%$ of the breast should receive $100 \% ; 3$ ) $\leq 3 \%$ of the $\mathrm{PD}$ to the entire volume of the contralateral breast; 4$)>30 \%$ of the PD administered to $<15 \%$ of the ipsilateral lung; $5)>5 \%$ of the PD to $<5 \%$ of cardiac volume for right breast lesions; 6 ) $>5 \%$ of the PD at $<40 \%$ of cardiac volume in left breast lesions.

\section{Discussion}

The association between ionizing radiation (IR) exposure and second malignancies has been well demonstrated in epidemiologic studies [10]. In particular, RIAS may be a rare but aggressive, late, secondary effect of breast radiotherapy. RIAS has been more frequently found in older women with a history of breast conservation surgery and radiation therapy since breast carcinoma commonly occurs in women between the ages of 55 and 69 [11]. Several mechanisms have been proposed to explain the physiopathological mechanisms underlying the development of RIAS. First, photons directly affect the DNA structure by inducing DNA breaks, particularly double-strand breaks. Secondary effects are the generation of reactive oxygen species (ROS) that oxidize proteins and lipids, but also induce additional damages to DNA, like generation of abasic sites and single strand breaks. All these findings may be responsible for genomic instability and cancerrelated genes mutation. About RIAS, several gene mutations have been reported in literature, some of which may be used to distinguish radiation-induced sarcomas from sporadic ones. Among these: the inactivation of the tumor suppressor gene p53, and the amplification of the 8q24 region containing the myc oncogene. Noteworthy, the prolonged cellular irritation ischemic breast and axillary tissues damaged by radiation-induced chronic lymphedema may interfere with the repair mechanisms, and promote tumorigenesis by increasing the amount of vascular growth factors within the tumor microenvironment. In this regard, FLT4 gene amplification (encoding VEGFR3) and KDR gene mutation (encoding VEGFR2) have been described to have a possible role in the development of RIAS. Finally, an association between breast cancer-related tumor-suppressor genes BRCA1/BRCA2 and RIAS may also exist, although the exact mechanism has yet to be established [12-15]. Histologically, RIAS usually presents with irregular, anastomosing vessels, lined by crowded swollen endothelial cells, with a scarce cytoplasm, showing different degrees of nuclear atypia. Typical immunohistochemical markers include CD31, CD34, factor VIII-related antigen, FLI1, ERG, and ulex europaeus 1 lectin [16-18]. RIAS has been reported to originate from the skin above the breast target volume, inside the radiation field. However, in some cases it may develop within the mammary parenchyma, arising from parenchymal vascular endothelial cells [19-22]. In our experience, 2 (0.67\%) patients developed RIAS after adjuvant APBI for early breast cancer out of 296 patients recruited as part of an Italian, multicenter, randomized, prospective trial 


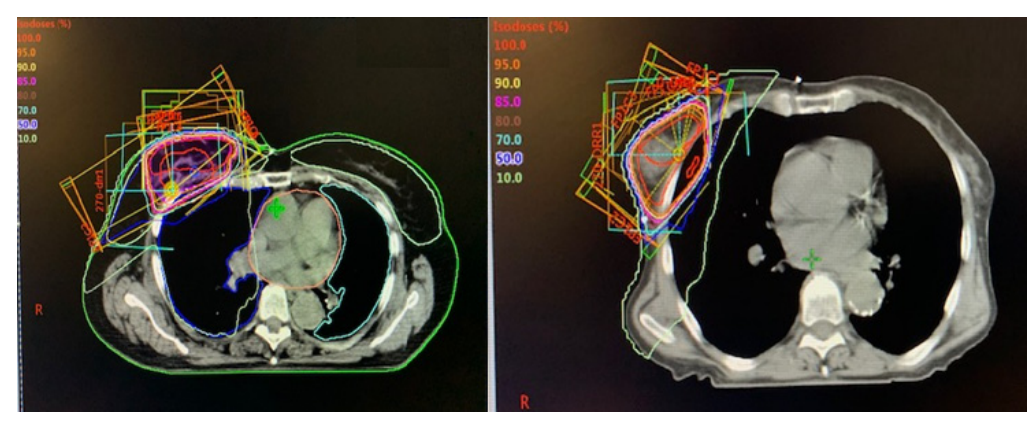

Figure 1. External beam radiation planning treatment for accelerated partial breast irradiation. A. Patient 1; B. Patient 2

(I.R.M.A Trial - NCT01803958) about efficacy and safety of APBI in early breast cancer treatment.

Patient 1 developed pericicatricial angiosarcoma originated from the skin inside the irradiation field, without evidence of mammary parenchymal involvement. In the second case, angiosarcoma developed far from the high-dose radiation site (that is, the tumor bed), in the lower quadrants, then it rapidly spread to all quadrants and finally involved the above skin and the entire, post-BSC, residual mammary parenchyma. In both cases, APBI was performed using a conformal, external beam radiation technique (3D-CRT), with 4 fields and $6 \mathrm{MV}$ photon beams (Fig. 1). The maximum dose to the whole residual breast volume was $40 \mathrm{~Gy}$ for Patient 1 and 40.3 Gy for Patient 2. In the second case, in the region in which angiosarcoma occurred, the average and maximum dose were $34 \mathrm{~Gy}$ and 39 Gy, respectively, and the volume corresponding to recurrence angiosarcoma was covered by $30 \%$ isodose (11.6 Gy). Indeed, it is still a matter of debate if the risk of RIAS is truly radiation-dose dependent.
To our knowledge, there are only two reported cases of RIAS after APBI, both as a consequence of treatment with MammoSite balloon Brachytherapy [23, 24]. Mansfield et al. [24] reported a case of a 75-year-old woman who developed RIAS with skeletal muscle invasion, in association with invasive ductal carcinoma, 8 years after partial breast treatment with MammoSite brachytherapy. RIAS originated close to the MammoSite applicator. Furthermore, the authors reported that an adjuvant radiotherapy of the chest wall was performed after mastectomy. Long-term survival data are not reported.

Andrews et al. [23] described a case of RIAS that occurred 74 months after MammoSite brachytherapy. Similarly, RIAS occurred close to the applicator field. In both cases, the administered dose was 34 Gy in twice-daily 10 fractions. Table 1 summarizes the main characteristics of the cases.

The latency for development of breast RIAS seems to be shorter when compared to radiationinduced sarcomas in general, with a reported aver-

Table 1. Summary of the main characteristics of the case series reported in literature

\begin{tabular}{|c|c|c|c|c|c|c|c|}
\hline Author & RT technique & $\begin{array}{l}\text { Radiation } \\
\text { dose }\end{array}$ & $\begin{array}{l}\text { Latency from } \\
\text { first and } \\
\text { second RT }\end{array}$ & $\begin{array}{l}\text { Salvage } \\
\text { treatment }\end{array}$ & $\begin{array}{c}\text { Adjuvant } \\
\text { radiotherapy }\end{array}$ & $\begin{array}{c}\text { Adjuvant } \\
\text { chemoterapy }\end{array}$ & Outcomes \\
\hline $\begin{array}{l}\text { Mansfield et al. } \\
\text { (2014) [24] }\end{array}$ & $\begin{array}{c}\text { MammoSite } \\
\text { brachytherapy }\end{array}$ & $\begin{array}{c}34 \text { Gy } \\
\text { in } 10 \text { fractions, } \\
\text { twice a day }\end{array}$ & 8 years & Mastectomy & Yes & Letrozole & $\begin{array}{l}\text { After } 5 \text { months no } \\
\text { signs of recurrence }\end{array}$ \\
\hline $\begin{array}{l}\text { Andrews et al. } \\
\text { (2010) [22] }\end{array}$ & $\begin{array}{c}\text { MammoSite } \\
\text { brachytherapy }\end{array}$ & $\begin{array}{c}34 \mathrm{~Gy} \\
\text { in } 10 \text { fractions, } \\
\text { twice a day }\end{array}$ & 6,1 years & Mastectomy & Unknown & Unknown & Unknown \\
\hline $\begin{array}{l}\text { Cozzi et al. } \\
\text { (2021) } \\
2 \text { patients }\end{array}$ & $\begin{array}{l}\text { 3DCRT with } \\
2 \text { tangential } \\
\text { coplanar } \\
\text { and two } \\
\text { non-coplanar }\end{array}$ & $\begin{array}{c}38.5 \text { Gy } \\
\text { in } 10 \text { fractions, } \\
\text { twice a day }\end{array}$ & $\begin{array}{c}\text { Both patients: } \\
8 \text { years }\end{array}$ & Mastectomy & $\begin{array}{c}\text { NO RT for } \\
\text { Both patients }\end{array}$ & $\begin{array}{l}\text { Patients 1: } \\
\text { none } \\
\text { Patients 2: } \\
\text { docetaxel and } \\
\text { gemcitabine }\end{array}$ & $\begin{array}{l}\text { Patients } 1 \text { : after } \\
3 \text { months no signs } \\
\text { of recurrence } \\
\text { Patients } 2 \text { : death } \\
1 \text { year after } \\
\text { the surgery }\end{array}$ \\
\hline
\end{tabular}


age of 10 to 12 years to develop after irradiation [25, 26]. It should be noted that in our patients and the case presented by Mansfield, RIAS occurred exactly eight years after radiotherapy treatment.

Due to its rarity, there are few scientific evidence regarding the management of RIAS. Mastectomy with negative margins is considered the standard of care. Negative margins are thought to be more important than the type of surgery itself for local control [27].

The role of chemotherapy has not been clearly established, and it is still object for study. Most of the available data originated from retrospective series or few case reports, and suggested that angiosarcomas are relatively sensitive to taxanes and anthracyclines [28].

The role of radiotherapy is controversial, instead, given the role of radiation in the pathogenesis of RIAS. Albeit with a low level of evidence, some studies reported an improved local control with re-irradiation after radical surgery. However, the available literature is too scarce to reach a conclusion $[29,30]$.

\section{Conclusion}

Radiation-induced angiosarcoma is a rare but aggressive type of cancer, which may occur as a secondary, late effect of breast irradiation after BSC. Actually, RIAS is a matter of debate, and many aspects about its origin and management are still to be better clarified or controversial. Due to its rarity, reports on such possible complication after APBI are still lacking in literature. To our knowledge, the present study is the first one to describe two events of RIAS after APBI using a 3D-CRT technique.

\section{Conflict of interest}

The authors declare no conflict of interest.

\section{Funding}

None declared.

\section{References}

1. Boyages J, Recht A, Connolly J, et al. Early breast cancer: predictors of breast recurrence for patients treated with conservative surgery and radiation therapy. Radiother Oncol. 1990; 19(1): 29-41, doi: 10.1016/0167-8140(90)90163q, indexed in Pubmed: 2173044.
2. Kurtz J, Amalric R, Brandone $\mathrm{H}$, et al. Local recurrence after breast-conserving surgery and radiotherapy. Frequency, time course, and prognosis. Cancer. 1989; 63(10): 19121917, doi: 10.1002/1097-0142(19890515)63:10<1912::aidcncr2820631007>3.0.co;2-y, indexed in Pubmed: 2702564.

3. Coles C, Griffin C, Kirby A, et al. Partial-breast radiotherapy after breast conservation surgery for patients with early breast cancer (UK IMPORT LOW trial): 5-year results from a multicentre, randomised, controlled, phase 3, non-inferiority trial. Lancet. 2017; 390(10099): 1048-1060, doi: 10.1016/s0140-6736(17)31145-5, indexed in Pubmed: 28779963.

4. Viani GA, Arruda CV, Faustino AC, et al. Partial-breast irradiation versus whole-breast radiotherapy for early breast cancer: A systematic review and update meta-analysis. Brachytherapy. 2020; 19(4): 491-498, doi: 10.1016/j. brachy.2020.03.003, indexed in Pubmed: 32340902.

5. Cohen-Hallaleh RB, Smith HG, Smith RC, et al. Radiation induced angiosarcoma of the breast: outcomes from a retrospective case series. Clin Sarcoma Res. 2017; 7: 15, doi: 10.1186/s13569-017-0081-7, indexed in Pubmed: 28794852.

6. Lyou Y, Barber E, Mehta R, et al. Radiation-Associated Angiosarcoma of the Breast: A Case Report and Literature Review. Case Rep Oncol. 2018; 11(1): 216-220, doi: 10.1159/000488314, indexed in Pubmed: 29805371.

7. Alves I, Marques JC. Radiation-induced angiosarcoma of the breast: a retrospective analysis of 15 years' experience at an oncology center. Radiol Bras. 2018; 51(5): 281-286, doi: 10.1590/0100-3984.2017.0129, indexed in Pubmed: 30369653.

8. Horisawa N, Adachi Y, Sawaki M, et al. A case of radiationassociated angiosarcoma after breast cancer. Surg Case Rep. 2018; 4(1): 131, doi: 10.1186/s40792-018-0538-9, indexed in Pubmed: 30406473.

9. Rombouts AJM, Huising J, Hugen N, et al. Assessment of Radiotherapy-Associated Angiosarcoma After Breast Cancer Treatment in a Dutch Population-Based Study. JAMA Oncol. 2019; 5(2): 267-269, doi: 10.1001/jamaoncol.2018.6643, indexed in Pubmed: 30676608.

10. Laé M, Lebel A, Hamel-Viard F, et al. Can cmyc amplification reliably discriminate postradiation from primary angiosarcoma of the breast? Cancer Radiother. 2015; 19(3): 168-174, doi: 10.1016/j.canrad.2015.01.001, indexed in Pubmed: 25863565.

11. Yang W, Hennessy B, Dryden M, et al. Mammary Angiosarcomas: Imaging Findings in 24 Patients. Radiology. 2007; 242(3): 725-734, doi: 10.1148/radiol.2423060163, indexed in Pubmed: 17325063.

12. Mentzel T, Schildhaus HU, Palmedo G, et al. Postradiation cutaneous angiosarcoma after treatment of breast carcinoma is characterized by MYC amplification in contrast to atypical vascular lesions after radiotherapy and control cases: clinicopathological, immunohistochemical and molecular analysis of 66 cases. Mod Pathol. 2012; 25(1): 75-85, doi: 10.1038/modpathol.2011.134, indexed in Pubmed: 21909081.

13. Shah S, Rosa M. Radiation-Associated Angiosarcoma of the Breast: Clinical and Pathologic Features. Arch Pathol Lab Med. 2016; 140(5): 477-481, doi: 10.5858/arpa.20140581-RS, indexed in Pubmed: 27128306. 
14. Torres KE, Ravi V, Kin K, et al. Long-term outcomes in patients with radiation-associated angiosarcomas of the breast following surgery and radiotherapy for breast cancer. Ann Surg Oncol. 2013; 20(4): 1267-1274, doi: 10.1245/ s10434-012-2755-y, indexed in Pubmed: 23224828.

15. Guo T, Zhang L, Chang NE, et al. Consistent MYC and FLT4 gene amplification in radiation-induced angiosarcoma but not in other radiation-associated atypical vascular lesions. Genes Chromosomes Cancer. 2011; 50(1): 25-33, doi: 10.1002/gcc.20827, indexed in Pubmed: 20949568.

16. Bonito FJ, de Almeida Cerejeira D, Dahlstedt-Ferreira C, et al. Radiation-induced angiosarcoma of the breast: A review. Breast J. 2020; 26(3): 458-463, doi: 10.1111/ tbj.13504, indexed in Pubmed: 31448482.

17. Zalaudek I, Gomez-Moyano E, Landi C, et al. Clinical, dermoscopic and histopathological features of spontaneous scalp or face and radiotherapy-induced angiosarcoma. Australas J Dermatol. 2013; 54(3): 201-207, doi: 10.1111/j.1440-0960.2012.00943.x, indexed in Pubmed: 22943153.

18. Selim A, Khachemoune A, Lockshin NA. Angiosarcoma: a case report and review of the literature. Cutis. 2005; 76(5): 313-317, indexed in Pubmed: 16422466.

19. Lehnhardt M, Bohm J, Hirsch T, et al. Radiation-induced angiosarcoma ofthe breast. Handchir Mikrochir Plast Chir. 2017; 49(2): 103-110.

20. Glazebrook KN, Magut MJ, Reynolds C, et al. Angiosarcoma of the breast. Am J Roentgenol. 2008; 57(4): 190-192.

21. Zemanova M, Rauova K, Boljesikova E, et al. Analysis of radiation-induced angiosarcoma of the breast. BratisI Lek Listy. 2014; 115(5): 307-310, doi: 10.4149/bll_2014_062, indexed in Pubmed: 25174061.

22. Hung J, Hiniker SM, Lucas DR, et al. Sporadic versus radiation-associatedangiosarcoma: a comparative clinicopathologic and molecular analysis of 48 cases. Sarcoma. 2013; 798403, doi: $10.1155 / 2013 / 798403$, indexed in Pubmed: 24082817.
23. Andrews S, Wilcoxon R, Benda Jo, et al. Angiosarcoma following MammoSite partial breast irradiation. Breast Cancer Res Treat. 2010; 124(1): 279-282, doi: 10.1007/ s10549-010-0948-2, indexed in Pubmed: 20496164.

24. Mansfield SA, Zynger DL, Agnese DM. Angiosarcoma and breast cancer recurrence eight years following mammosite therapy. Breast J. 2014; 20(6): 658-660, doi: 10.1111/tbj.12337, indexed in Pubmed: 25228265.

25. Farran Y, Padilla O, Chambers K, et al. Atypical Presentation of Radiation-Associated Breast Angiosarcoma: A Case Report and Review of Literature. Am J Case Rep. 2017; 18: 1347-1350, doi: 10.12659/ajcr.905157, indexed in Pubmed: 29249796.

26. Cuperus $E$, Leguit R, Albregts $M$, et al. Post radiation skin tumors: basal cell carcinomas, squamous cell carcinomas and angiosarcomas. A review of this late effect of radiotherapy. Eur J Dermatol.2013;23(6): 749-757, doi: 10.1684/ ejd.2013.2106, indexed in Pubmed: 24153098.

27. Lindford A, Böhling T, Vaalavirta L, et al. Surgical management of radiation-associated cutaneous breast angiosarcoma. J Plast Reconstr Aesthet Surg. 2011; 64(8): 1036-1042, doi: 10.1016/j.bjps.2011.02.014, indexed in Pubmed: 21377947.

28. Skubitz K, Haddad P. Paclitaxel and pegylated-liposomal doxorubicin are both active in angiosarcoma. Cancer. 2005; 104(2): 361-366, doi: 10.1002/cncr.21140, indexed in Pubmed: 15948172.

29. Smith TL, Morris CG, Mendenhall NP. Angiosarcoma after breast-conserving therapy: long-term disease control and late effects with hyperfractionated accelerated re-irradiation (HART). Acta Oncol. 2014; 53(2): 235-241, doi: 10.310 9/0284186X.2013.819117, indexed in Pubmed: 24345278.

30. Billings SD, McKenney JK, Folpe AL, et al. Cutaneous angiosarcoma following breast-conserving surgery and radiation: an analysis of 27 cases. Am J Surg Pathol. 2004; 28(6): 781-788, doi: 10.1097/01.pas.0000126055.33916.0b, indexed in Pubmed: 15166670. 